\title{
Retrospective study on laparoscopic hysterectomy by vaginal vault closure method in a tertiary care centre
}

\author{
Pradeep Ganiga*, Divyashree B.
}

Department of Obstetrics and Gynecology, A. J. Institute of Medical Sciences and Research Centre, Mangalore, India

Received: 28 May 2018

Accepted: 04 June 2018

*Correspondence:

Dr. Pradeep Ganiga,

E-mail: pradeepganiga104@gmail.com

Copyright: (C) the author(s), publisher and licensee Medip Academy. This is an open-access article distributed under the terms of the Creative Commons Attribution Non-Commercial License, which permits unrestricted non-commercial use, distribution, and reproduction in any medium, provided the original work is properly cited.

\begin{abstract}
Background: Hysterectomy is the frequently performed operation for benign conditions of uterus, but the route of hysterectomy is always a matter of discussion. Laparoscopic hysterectomy is an expensive and time-consuming procedure and could be associated with increased risk of thermal damage related injuries to the surrounding structures. Presently, laparoscopic hysterectomy is considered to be a day care surgery because of technological improvement in electro-surgical unit and expertise of doing surgery. The objective of this study was as a retrospective observational study conducted in teaching hospital to study the factors like age, parity, indication and procedure related complications in detail.

Methods: A total number of 40 patients who have undergone laparoscopic hysterectomy for various indications with vault closure by vaginal method with no. 1 PDS. A detailed analysis of cases was done according to age, parity, indication, intra-operative and post-operative outcomes.

Results: Fibroid uterus was the most common indication for laparoscopic hysterectomy. Overall postoperative complication rate was $22.5 \%$ with no major morbidity reported.

Conclusions: Laparoscopic hysterectomy has to be done with proper selection of patient and good peri-operative care. Vault closure by vaginal method has significantly reduced operating time, without increasing the morbidity.
\end{abstract}

Keywords: Electro-surgical unit, Laparoscopic hysterectomy, Parity index, Vaginal vault

\section{INTRODUCTION}

Hysterectomy remains the most common major gynaecological operations done worldwide. It may be carried out by three different routes and its variations, vaginal, abdominal and laparoscopy with the advancement of laparoscopic technology, equipment, and training hysterectomies are increasingly performed laparoscopically. ${ }^{1}$ The first laparoscopic hysterectomy was performed in the year 1989 by Reich et al. ${ }^{2}$

The laparoscopic hysterectomy has become one of the popular methods of hysterectomy in view of decreased morbidity, rapid, recovery and early resumption of work. One of the main drawbacks of laparoscopy is the steep learning curve and difficulty in improving the expertise. The advancement in the bio-medical field has made laparoscopic hysterectomy a surgeon friendly operation because of improvement in the electro-surgical delivery system. The various energy systems commonly used in the laparoscopic procedures are, monopolar, bipolar and ultrasonic current with the added disadvantage of lateral spread to the surrounding structure mainly to the ureter and bowel. Compared to monopolar and bipolar current, the ultrasonic has minimal lateral spread of energy to the surrounding structure which makes it more acceptable procedure for laparoscopic hysterectomy. The effective coagulation will not be satisfactory in ultrasonic current as compared to bipolar current, because of this reason bipolar energy has become effective in coagulating the 
vessels, mainly the uterine artery which has a diameter of $7 \mathrm{~mm}$.

There is lot of controversy among gynae-laparoscopists about the usage of monopolar currents mainly for cutting the vault of the vagina. Many studies have proved that monopolar cutting of the vault will increase the intraoperative bleeding, increased risk of uro-genital thermal injuries and increased post-operative discharge per vaginum. A Cochrane database review by Johnson et al, suggests that TLH should be preferred to abdominal hysterectomy for benign gynaecological disease. ${ }^{3}$

Sexual function and quality of life have been the focus of discussion after hysterectomy in recent years in view of sexually active women undergoing hysterectomy for various reasons. However, studies investigating postoperative psychological and sexual effects are limited.

Uterus delivery was done either vaginally or by morcellation. Both methods have advantages and disadvantages depending on the availability and expertise of the surgeon. The closure of the vault can also be done by intra-corporeal suturing or by vaginal method, both are equally advantageous especially in preventing future vault prolapse by securing utero-sacral ligament to the vault of vagina.

\section{METHODS}

The study is a retrospective study conducted in gynaecology department of a tertiary care hospital, AJIMS ana RC, Mangaluru. A total number of 40 cases had undergone laparoscopic hysterectomy between 2016 to 2018 for various indications.

The patients who had undergone laparoscopic hysterectomy after counselling with detailed explanation about advantages and disadvantages of the procedure in their understandable language were included in the study. the surgeries were done by a single surgeon. In all cases after laparoscopic hysterectomy the vault was closed by vaginal method with No.1 PDS.

The data regarding age, parity, indication of surgery, size of the uterus, intraoperative and postoperative complications were noted. All patients were given peglec enema day before surgery for bowel preparation. All the surgeries were done in general anaesthesia in modified lithotomy position.

Pre-operative antibiotics were given to all the patients as per the hospital protocol. Surgeries were done by using bipolar forceps and vessel sealer. Pneumoperitoneum was created by Veress needle insertion, followed by Primary trocar insertion, 1 inch above the umbilicus.

Myoma screw was used for uterine manipulation for all the cases. Uterus was delivered by vaginal route in all cases (morcellator was not used). Vault closure was done through the vagina by using No. 1 PDS. All the intraoperative complications like injury to the surrounding structure, bleeding etc., were noted. Post-operative complications like distension of the abdomen, blood stained urine, fever, port site infection, bleeding per vaginum were noted. Majority of the cases were discharged on $4^{\text {th }}$ post-operative day after passing stools.

\section{Inclusion criteria}

- No history of previous surgeries

- Uterus size of less than 16 weeks

- Cases with benign pathology.

\section{RESULTS}

Data of total 40 cases who have undergone laparoscopic hysterectomy were noted and was analysed.

Table 1: Age distribution of cases.

\begin{tabular}{|ll|}
\hline Age & Number of patients \\
\hline $35-40$ & $9(22.5 \%)$ \\
\hline $40-45$ & $13(32.5 \%)$ \\
\hline $45-50$ & $14(35 \%)$ \\
\hline$>50$ & $4(10 \%)$ \\
\hline
\end{tabular}

All over the world, most of the hysterectomies are done in the peri-menopausal or post-menopausal age either because of severity of symptoms or because of premalignant conditions like endometrial hyperplasia with atypia.

As per the literature, the fibroids are the most common indication for hysterectomy in peri-menopausal age group in view of increase in the symptoms like bleeding per vaginum. In present study, the most common age group of patients was between 45-50 years followed by 40-45 years $(32.5 \%)$.

Table 2: Distribution of cases according to parity.

\begin{tabular}{|ll|}
\hline Parity index & Number of patients \\
\hline Para 1 & $5(12.5 \%)$ \\
\hline Para 2 & $20(50 \%)$ \\
\hline Para 3 & $11(27.5 \%)$ \\
\hline Para 4 & $3(7.5 \%)$ \\
\hline Nulliparous & $1(2.5 \%)$ \\
\hline
\end{tabular}

The parity has a role in doing procedures like hysterectomy because of the laxity of ligaments which makes the procedure comparatively faster and easy for the operating surgeon.

In present study majority of patients were in the group of para two, which accounts to $50 \%$. Only one patient was nulliparous which generally takes longer duration when compared to parous women. 
Table 3: Distribution of cases according to presenting symptoms.

\begin{tabular}{|ll|}
\hline Symptoms & Number of patients \\
\hline Polymenorrhea & $2(5 \%)$ \\
\hline Menorrhagia & $30(75 \%)$ \\
\hline Dysmenorrhea & $2(5 \%)$ \\
\hline Abdominal pain & $3(7.5 \%)$ \\
\hline Post-menopausal bleeding & $3(7.5 \%)$ \\
\hline
\end{tabular}

All over the world, menorrhagia has become the commonest problem making the women to undergo uterus removal surgery, especially in peri-menopausal and post-menopausal age with different benign diseases.

In present retrospective study authors found that $75 \%$ of patients who had undergone laparoscopic hysterectomy for menorrhagia, followed by abdominal pain and postmenopausal bleeding with $7.5 \%$ each. Severe dysmenorrhea was the presenting complaint for $5 \%$ of cases of laparoscopic hysterectomy most likely to be adenomyosis of the uterus.

Table 4: Distribution of patients as per endometrial thickness.

\begin{tabular}{|ll|}
\hline Endometrial thickness & Number of patients \\
\hline$<4 \mathrm{~mm}$ & 0 \\
\hline $4-6 \mathrm{~mm}$ & $10(25 \%)$ \\
\hline$>6 \mathrm{~mm}$ & $30(75 \%)$ \\
\hline
\end{tabular}

The ultrasound assessment of endometrial thickness is one of the important component of pre-operative assessment of all cases who are planned for hysterectomy. In present study, majority i.e. $75 \%$ of patients had 4-6mm of endometrial thickness which directly indicates benign nature of the diseases for which hysterectomy was planned.

Table 5: Distribution of patients according to preoperative diagnosis.

\begin{tabular}{|ll|}
\hline Pre-operative diagnosis & Number of patients \\
\hline Fibroid & $12(30 \%)$ \\
\hline Adenomyosis & $21(52.5 \%)$ \\
\hline $\begin{array}{l}\text { Atypical endometrial } \\
\text { hyperplasia }\end{array}$ & $6(15 \%)$ \\
\hline
\end{tabular}

In present study all the patients were taken with preoperative diagnosis after taking endometrial biopsy in indicated cases.

It is showing that majority of the cases were fibroid uterus $(52.5 \%)$, followed by abnormal uterine bleeding $(30 \%)$ cases. One case with the histopathological diagnosis of atypical endometrial hyperplasia was operated but post-operative diagnosis revealed early endometrial cancer.
Table 6: Distribution of cases according to prophylactic salphingo- oophorectomy status.

\begin{tabular}{|ll|}
\hline $\begin{array}{l}\text { Prophylactic salphingo- } \\
\text { oophorectomy status }\end{array}$ & Number of patients \\
\hline $\begin{array}{l}\text { Bilateral prophylactic } \\
\text { oophorectomy done }\end{array}$ & $16(40 \%)$ \\
\hline $\begin{array}{l}\text { Not undergone bilateral } \\
\text { prophylactic oophorectomy }\end{array}$ & $24(60 \%)$ \\
\hline
\end{tabular}

The prophylactic bilateral prophylactic oophorectomy was done in patients after proper counselling and obtaining consent in $40 \%$ of patients. The patients who had undergone prophylactic oophorectomy were informed about the occurrence of menopausal symptoms and were advised for hormonal replacement therapy depending on the patient's severity of symptoms.

Table 7: Distribution of patients according to postoperative complications.

\begin{tabular}{|ll|}
\hline $\begin{array}{l}\text { Post-operative } \\
\text { complications }\end{array}$ & Number of patients \\
\hline Fever & $2(5 \%)$ \\
\hline Distension & $1(2.5 \%)$ \\
\hline Bleeding per vaginum & $1(2.5 \%)$ \\
\hline Vault Infection & $2(5 \%)$ \\
\hline UTI & $3(7.5 \%)$ \\
\hline No complications & $31(77.5 \%)$ \\
\hline
\end{tabular}

Patients were monitored to detect post-operative complications like infection, haemorrhage, UTI etc., each complication was noted and managed further. In present study, the post-operative complications are minimal in view of proper selection of patients and adequate postoperative care, $7.5 \%$ of the patients had UTI and $5 \%$ of the patients had fever. Only one of the patients out of 40 was readmitted with bleeding per vaginum for one month, diagnosed as vault infection, and responded well to injectable antibiotics and antifibrinolytic therapy.

\section{DISCUSSION}

Present study was a retrospective study which included 40 cases of laparoscopic hysterectomy. Laparoscopic hysterectomy is currently accepted as a safe and efficient way to manage benign uterine pathology in selected patients. ${ }^{4}$ However, the challenges and limitations of this procedure are still debatable. ${ }^{5}$ The outcome of laparoscopic hysterectomy and the technique used in performing the operation well depend on various factors, such as the indication for hysterectomy, associated comorbidity, surgeon's experience and availability of the necessary equipment.

In present study, as expected two-thirds of patients were between 40 and 50 years. A study conducted by Saleh $\mathrm{MM}$ et al reported that mean age of patients who are undergoing laparoscopic hysterectomy was 44 years which similar to present study report. ${ }^{6}$ 
Present study revealed that, $50 \%$ of the patients were para-two. A study conducted by Perino A et al, reported that the mean parity was 2.3 , which might have a direct impact on the duration of the surgery. ${ }^{7}$

Similar to other studies, present study revealed that fibroid uterus $(52.5 \%)$ is the most common indication for laparoscopic hysterectomy. A study by Pattnaik $\mathrm{T}$ et al reported that most common indication for laparoscopic hysterectomy was fibroid uterus (40.2\% of cases), followed by AUB (28.32\%) which is similar to what was reported in present study $(30 \%) .{ }^{8}$ Another comparative study between abdominal and laparoscopic hysterectomy by Meryem et al reported that $19.5 \%$ of total patients of laparoscopic hysterectomy was fibroid uterus and $26.8 \%$ of patients of abdominal hysterectomy had fibroid uterus. ${ }^{9}$

In present analysis the post-operative complications were minimal which is mainly attributed to the selection of the cases like, no previous history of abdominal surgeries. In a study conducted by Donnez et al for a series of 400 laparoscopic hysterectomies for benign disease and reported that no minor or major complications were reported except bladder injury which was $<2 \mathrm{~cm}$ in 3 cases and in the same study the total major complications reported $1.59 \%$ of cases. ${ }^{10}$ In present study, the overall complications were $22.5 \%$ which is similar to a study conducted by Hill DJ et al concluded that $15.9 \%$ had complications. ${ }^{11}$ Another study reported in 2016 by Wang $\mathrm{H}$ where he compared removal of uterus either by trans vaginal route or morcellation, pelvic haematoma incidence was higher in trans-vaginal group (1.2\%), compared to morcellation group $(0.4 \%)$, but in present study none of the cases had pelvic haematoma where authors delivered uterus by trans-vaginal route, in the same study vaginal stump infection was more in morcellation group $(2.4 \%)$ compared to $1.9 \%$ in transvaginal group. ${ }^{12}$ Another study by David $\mathrm{E}$ et al compared laparoscopic hysterectomy versus laparoscopic assisted vaginal hysterectomy and reported that overall complications which includes intra-operative and postoperative complications which is similar in both the groups. ${ }^{13}$

A study conducted on post-operative pain comparing laparoscopic and vaginal hysterectomy by Marika $\mathbf{J}$ et al concluded that post-operative pain in first hours is more intensive after vaginal hysterectomy and lasts longer than after laparoscopic hysterectomy but is similar in 24 hours. ${ }^{14}$

\section{CONCLUSION}

Laparoscopic hysterectomy can be done in any benign indication. The mainstay of procedure is proper selection of the patient, good preoperative preparation and postoperative care. Vault closure by vaginal method is as equally good as intra-corporeal suturing. The main advantage of vaginal vault closure is that it is less time consuming, especially for surgeons in the early part of the learning curve. Overall complication rate by vaginal or intra-corporeal method is equal.

Funding: No funding sources

Conflict of interest: None declared

Ethical approval: The study was approved by the Institutional Ethics Committee

\section{REFERENCES}

1. Kavallaris A, Chalvatzas N, Kelling K, Bohlmann MK, Diedrich K, Hornemann A. Total laparoscopic hysterectomy without uterine manipulator: description of a new technique and its outcome. Arch Gynecol Obstet. 2011 May 1;283(5):1053-7.

2. Reich H, DeCAPRIO JO, McGLYNN FR. Laparoscopic hysterectomy. J Gynecol Surgery. 1989;5(2):213-6.

3. Nieboer TE, Johnson N, Lethaby A, Tavender E, Curr E, Garry R, et al. Surgical approach to hysterectomy for benign gynaecological disease. Cochrane database syst rev. 2009 Jul 8;3(8).

4. Wattiez A, Soriano D, Cohen SB, Nervo P, Canis M, Botchorishvili R, et al. The learning curve of total laparoscopic hysterectomy: comparative analysis of 1647 cases. The J Am Assoc Gynecol Laparoscopists. 2002 Aug 1;9(3):339-45.

5. Mettler L, Ahmed-Ebbiary N, Schollmeyer T. Laparoscopic hysterectomy: challenges and limitations. Minimally Invasive Therapy Allied Technologies. 2005 Jun 1;14(3):145-59.

6. Saleh MM, Seoud AA, Zaklama MS. Challenges of laparoscopic hysterectomy: a 10-year experience in UK hospitals. Gynecol Surgery. 2008 May $1 ; 5(2): 115-20$.

7. Perino A, Cucinella G, Venezia R, Castelli A, Cittadini E. Total laparoscopic hysterectomy versus total abdominal hysterectomy: an assessment of the learning curve in a prospective randomized study. Human Reprod. 1999 Dec 1;14(12):2996-9.

8. Pattanaik T, Mishra SP, Das S. Total Laparoscopic Hysterectomy-A Retrospective Observational Study in a Teaching Hospital.

9. Eken MK, İlhan G, Temizkan O, Çelik EE, Herkiloğlu D, Karateke A. The impact of abdominal and laparoscopic hysterectomies on women's sexuality and psychological condition. Turkish J Obstet Gynecol. 2016 Dec;13(4):196.

10. Donnez O, Donnez J. A series of 400 laparoscopic hysterectomies for benign disease: a single centre, single surgeon prospective study of complications confirming previous retrospective study. BJOG: An I J Obstet Gynaecol. 2010 May 1;117(6):752-5.

11. Hill DJ, Maher PJ, Wood CE, Lolatgis N, Lawrence A, Dowling B, et al. Complications of laparoscopic hysterectomy. The J Am Assoc Gynecol Laparoscopists. 1994 Feb 1;1(2):159-62.

12. Wang H, Li P, Li X, Gao L, Lu C, Zhao J, et al. Total laparoscopic hysterectomy in patients with 
large uteri: comparison of uterine removal by transvaginal and uterine morcellation approaches. BioMed Research International. 2016;2016.

13. David-Montefiore E, Rouzier R, Chapron C, Darai E, Collegiale d'Obstétrique et Gynécologie de Paris-Ile de France. Surgical routes and complications of hysterectomy for benign disorders: a prospective observational study in French university hospitals. Human Reprod. 2007 Jan 1;22(1):260-5.
14. Arsen JM. Comparative Analysis of Laparoscopic and Vaginal Hysterectomy-Postoperative Pain. J Universal Surgery. 2017 Mar 30;5(2).

Cite this article as: Ganiga $P$, Divyashree $B$.

Retrospective study on laparoscopic hysterectomy by vaginal vault closure method in a tertiary care centre. Int J Reprod Contracept Obstet Gynecol 2018;7:2634-8. 